\title{
Structural and functional asymmetry of the neonatal cerebral cortex
}

\author{
Logan Z. J. Williams $s^{\mathrm{a}, \mathrm{b} \otimes}$, Sean P. Fitzgibbon ${ }^{\mathrm{c}}$, Jelena Bozek ${ }^{\mathrm{d}}$, Anderson M. Winkler ${ }^{\mathrm{e}}$, Ralica Dimitrova ${ }^{\mathrm{a}}$, Tanya Poppe ${ }^{\mathrm{a}}$, \\ Andreas Schuh ${ }^{\mathrm{f}}$, Antonios Makropoulos ${ }^{\mathrm{a}}$, John Cupitt ${ }^{\mathrm{f}}$, Jonathan O'Muircheartaigh ${ }^{\mathrm{a}, \mathrm{g}, \mathrm{h}}$, Eugene P. Duff ${ }^{\mathrm{c}}$, Lucilio \\ Cordero-Grande ${ }^{\mathrm{a}, \mathrm{i}}$, Anthony N. Price ${ }^{\mathrm{a}}$, Joseph V. Hajnal ${ }^{\mathrm{a}}$, Daniel Rueckert ${ }^{\mathrm{f}, \mathrm{j}}$, Stephen M. Smith $^{\mathrm{c}}$, A. David Edwards $^{\mathrm{a}, \mathrm{g}, \mathrm{h}}$, and \\ Emma C. Robinson ${ }^{\mathrm{a}, \mathrm{b} \otimes}$ \\ ${ }^{a}$ Centre for the Developing Brain, Department of Perinatal Imaging and Health, School of Biomedical Engineering and Imaging Sciences, King's College London, London, \\ SE1 7EH, UK \\ ${ }^{b}$ Department of Biomedical Engineering, School of Biomedical Engineering and Imaging Science, King's College London, London, SE1 7EH, UK \\ ${ }^{c}$ Centre for Functional MRI of the Brain (FMRIB), Wellcome Centre for Integrative Neuroimaging, Nuffield Department of Clinical Neurosciences, University of Oxford. John \\ Radcliffe Hospital, Oxford, OX3 9DU, UK \\ ${ }^{\mathrm{d}}$ Faculty of Electrical Engineering and Computing, University of Zagreb, Zagreb, Croatia \\ Emotion and Development Branch, National Institute of Mental Health, National Institutes of Health, Bethesda, Maryland, USA \\ ${ }^{\mathrm{f}}$ Department of Computing, Imperial College London, London SW7 2RH, UK \\ ${ }^{g}$ Department for Forensic and Neurodevelopmental Sciences, Institute of Psychiatry, Psychology and Neuroscience, King's College London, London, SE5 8AF, UK \\ ${ }^{\mathrm{h}} \mathrm{MRC}$ Centre for Neurodevelopmental Disorders, King's College London, London, SE1 1UL, UK \\ ${ }^{i}$ Biomedical Image Technologies, ETSI, Telecomunicación, Universidad Politécnica de Madrid and CIBER-BBN, Madrid, 28040, Spain \\ ${ }_{\mathrm{j}}^{\mathrm{K}}$ linikum rechts der Isar, Technical University of Munich, Munich, Germany
}

Features of brain asymmetry have been implicated in a broad range of cognitive processes; however, their origins are still poorly understood. Using a new left-right symmetric, spatiotemporal cortical surface atlas, we investigated cortical asymmetries in $\mathbf{4 4 2}$ healthy newborn infants soon after birth, using structural and functional magnetic resonance images from the Developing Human Connectome Project. We identified previously unrecognised structural and functional asymmetries in auditory, visual and sensorimotor cortices, which closely resemble known asymmetries in adults. These findings show that cortical asymmetries are largely determined prenatally and suggest that they may constrain the development of lateralised functions in later life. In adults, deviations in brain asymmetry have been implicated in a broad range of developmental and psychiatric disorders, some of which have been associated with abnormal perinatal neurodevelopment. To test the hypothesis that normal cortical asymmetry is disrupted in the perinatal period by severe environmental stress, we compared cortical asymmetries between the same group of term neonates and 103 preterm neonates imaged at term-equivalent age. No significant differences were seen between these two cohorts, showing that the development of cortical asymmetries proceeds largely unaffected by preterm birth.

developing human connectome project | cortical surface | asymmetry | resting-state networks | preterm birth

Correspondence: logan.williams@kcl.ac.uk; emma.robinson@kcl.ac.uk

\section{Introduction}

Asymmetry is an important feature of human brain organisation (1), implicated in a number of cognitive processes including language $(2,3)$ and face processing (4). Moreover, changes to typical brain asymmetry have been associated with neurodevelopmental and psychiatric disorders including autism spectrum disorder $(5,6)$, schizophrenia $(7,8)$, obsessive-compulsive disorder (9), attention-deficit hyperactivity disorder (10), and dyslexia (11). However, the origins of these asymmetries (12) and the factors affecting their development (13) remain poorly understood.

Using magnetic resonance imaging (MRI) to characterise cortical asymmetries in the neonatal period can help clarify their ontogenesis (14). Previous work has investigated structural (15-22) and functional (23-25) asymmetries in the perinatal period and early infancy. As is seen in adults (20), term and preterm neonatal brains exhibit leftward volume (left greater than right) and sulcal depth (left deeper than right) asymmetry $(19,20,22)$ in regions important for processing language. Functional asymmetries, measured using taskbased functional MRI (fMRI), are also present at birth and during infancy, and include lateralised responses to speech $(25,26)$ and music $(23)$.

Although these studies have greatly advanced our understanding of brain asymmetry in early life, they have important limitations. Volumetric-based analyses (15, 22-25) fail to capture the structural and functional heterogeneity of the cortex, due to residual misalignment between cortical folds during registration $(27,28)$. In the case of fMRI, this leads to leakage of blood-oxygen-level-dependent signal across adjacent cortical folds (29). Additionally, previous surfacebased approaches register individual subject cortical surfaces to a single group template directly from their native space $(19,20)$. This is undesirable as the deformations required for registration increase as the age difference between the subject and the template increases, to properly account for the marked changes in cortical morphology that characterise perinatal brain development (30-32).

The Developing Human Connectome Project (dHCP) is an open-science project, which has advanced image acquisition and processing to produce a large cross-sectional set of multimodal MRI data in neonates between 24-45 weeks' postmenstrual age (PMA). Here we present a new left-right symmetric, surface-based spatiotemporal atlas that models population average changes in cortical morphology between 28-44 weeks' PMA. After surface-based registration to this new template, we investigated structural asymmetry in 442 healthy term-born neonates at a vertex-wise level using permutation testing. As there are only a limited number of studies directly testing for functional asymmetry in the neona- 
tal period (33), we also investigated asymmetries of intrinsic cortical activity using resting-state fMRI (rs-fMRI) mapped to the cortical surface.

Finally, it is well known that neurodevelopment in preterm neonates (born $<37$ weeks' gestational age (GA)) is characterised by dysmaturation $(34,35)$, and that those born preterm have poorer long-term developmental and behavioural outcomes compared to their term-born counterparts (36-39). By comparing cortical asymmetries between the same 442 termborn neonates and 103 preterm neonates at term-equivalent age (TEA), we directly tested the impact of early life environmental perturbations on the development of structural and functional asymmetry in the newborn brain.

\section{Results}

\section{Structural Asymmetries in the Healthy Term Neonatal}

Brain. Significant regions of sulcal depth, surface area and cortical thickness asymmetry are shown in Figure 1a, with corresponding $t$-statistic maps in Figure S1a.

Asymmetries were observed in several sulci on the lateral and medial hemispheric surface. On the lateral surface, leftward asymmetry (left deeper than right) was observed for the anterior and posterior lateral fissure. There was also rightward asymmetry (right deeper than left) of the middle portion of the superior temporal sulcus. On the medial hemispheric surface, there was leftward asymmetry of the calcarine sulcus and rightward asymmetry of the parieto-occipital sulcus.

Regarding surface area, on the lateral hemispheric surface there were leftward asymmetries (left $>$ right) located in the anterior lateral fissure, supramarginal, postcentral, and inferior temporal gyri, and occipital pole. There were rightward asymmetries (right $>$ left) located in the superior and middle temporal, rostral middle frontal, and inferior frontal gyri, and insula. On the medial hemispheric surface, there were leftward asymmetries in the isthmus of the cingulate gyrus and rostral anterior cingulate gyrus. There were also rightward asymmetries in the parieto-occipital sulcus, lingual and caudal anterior cingulate gyri.

For cortical thickness, on the lateral hemispheric surface there were leftward asymmetries (left $>$ right) located in the insula, posterior lateral fissure, supramarginal and angular gyri, lateral aspect of the central sulcus, pre- and postcentral gyri, and occipital pole. There were also rightward asymmetries (right $>$ left) located in the superior and middle temporal gyri. On the medial surface, there were rightward asymmetries located in the calcarine and parieto-occipital sulci, precuneus and caudal aspect of the superior frontal gyrus.

\section{Functional Asymmetry in the Healthy Term Neonatal}

Brain. Group independent component analysis (ICA) on the cortical surface generated 11 resting-state networks (RSNs), which are shown in Figure S2. Significant asymmetries were found in seven of these RSNs (Figure 1b), with corresponding $t$-statistic maps in Figure S1b.

In the medial motor RSN, a leftward asymmetric region was located in the dorsolateral aspect of the central sulcus, and a rightward asymmetric region in the dorsomedial aspect of the central sulcus. The lateral motor RSN had a leftward asymmetric region located in the midportion of the central sulcus and a rightward asymmetric region located in the inferolateral aspect of the central sulcus. For the somatosensory RSN, the leftward asymmetric region was located in the midportion of the postcentral gyrus, and the rightward asymmetric region in the inferolateral aspect of the postcentral gyrus. A single rightward asymmetric region was located in the dorsomedial aspect of the precentral gyrus in the motor association RSN. Asymmetry in the auditory RSN comprised a leftward asymmetric region located along the anterior end of the superior temporal gyrus and a rightward asymmetric region at the posterior end of the superior temporal gyrus and lateral fissure. The visual RSN consisted of a single rightward asymmetric region located on the medial aspect of the occipital lobe. Rightward asymmetry in the parietal RSN was localised to the subparietal sulcus and dorsal aspect of the precuneus.

To test if residual differences in individual sulcal depth asymmetry affected functional asymmetry, we performed an additional experiment where single subject sulcal depth asymmetry maps were included as a per-vertex explanatory variable (see Methods: Statistical Analysis). Residual sulcal depth asymmetries had minimal impact on the spatial extent and significance of regions of functional asymmetry (Figures S3 and S4).

Effect of Preterm Birth. There were no significant differences in structural or functional asymmetries between preterm- and term-born neonates at TEA. Corresponding $p$ value and $t$-statistic spatial maps are shown in Figures S5 and S6, respectively.

\section{Discussion}

This paper details the largest investigation into the development of cortical asymmetries in the newborn brain to date; the first to simultaneously investigate asymmetries in cortical morphology and intrinsic cortical activity (as assessed with rs-fMRI); and the first to directly measure the impact of preterm birth on their development. The assessment of cortical asymmetries was contingent on accurate correspondence of features between hemispheres and across individuals, which was achieved through: 1) development of a novel left-right symmetric, spatiotemporal cortical surface atlas (based on work described in (32) and (40)), and 2) biomechanically-constrained surface registration to this template using Multimodal Surface Matching (MSM) (41, 42) and template-to-template registration (30).

Our findings are consistent with previous volumetric-based studies in neonates and adults investigating structural and functional asymmetry. We replicated rightward asymmetry of the middle portion of the superior temporal sulcus, which has been demonstrated in fetuses $(16,17)$, preterm and term neonates (18-20,43), infants (21), children (44) and adults (45). In addition, patterns of cortical surface area and thickness seen here closely resemble those seen in adults (46), as surface area asymmetries were consistent in the superior and middle temporal gyri, inferior and middle frontal gyri, 


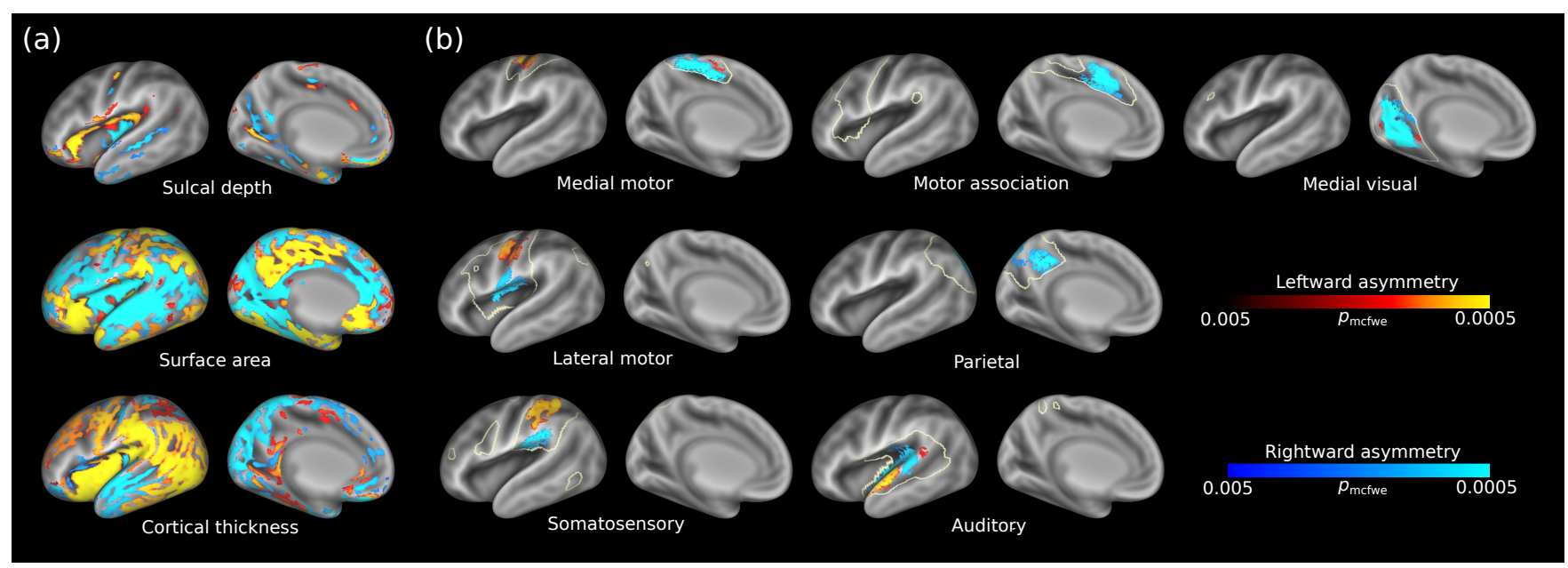

Fig. 1. Structural (a) and functional (b) asymmetries in the healthy term-born neonatal cortex at term-equivalent age. Leftward asymmetries are represented by the red-yellow colour scale and rightward asymmetries by the blue-light blue colour scale. Significantly asymmetric regions are visualised on a very inflated 40-week PMA left hemispheric surface, overlaid on a 40week PMA sulcal depth template (grey scale colour scheme). Off-white lines surrounding the functional asymmetries represent the mask used to threshold single subject asymmetry maps (see Methods: Generating Asymmetry Maps). Data at https: //balsa.wustl.edu/wNlvv.

inferior postcentral gyrus, supramarginal gyrus, caudal anterior and posterior cingulate gyri, medial occipital lobe, precuneus, and anterior insula; and cortical thickness asymmetries were consistent in the superior and middle temporal gyri, middle and superior frontal gyri, and precentral gyrus (46).

Although functional asymmetries have not previously been investigated using surface-based analyses, our findings are consistent with volumetric-based studies in neonates and adults. Studies investigating language using speech stimuli in task-based fMRI have shown leftward asymmetric activation in the primary auditory cortex of infants $(25,26)$, children (47) and adults $(2,3)$. On the other hand, studies in neonates have shown that music evoked predominantly right-hemispheric activation in primary and association auditory cortices (23). These findings are consistent with studies in adults demonstrating that the left auditory cortex is specialised for resolving temporal changes (for instance, discriminating between syllables), whilst the right auditory cortex is specialised for resolving changes in pitch (48-50). We also demonstrated, for the first time using rs-fMRI, rightward asymmetry of the visual network in the neonatal period. This is consistent with volumetric-based functional asymmetry studies in adults that show rightward asymmetry of primary and association visual networks $(51,52)$.

The marked similarity in structural and functional asymmetries between neonates and adults suggest that cortical asymmetries are broadly conserved. It is possible that such conservation is, at least in part, genetically determined. Recent work investigating the genomic loci underpinning brain asymmetries has implicated genes that are expressed during prenatal brain development (53), which are involved in molecular pathways responsible for left-right axis patterning in vertebrates (53), visual and auditory pathway development (54), neural activity during development of the so- matosensory cortex (55), and regulation of axonal guidance and synaptogenesis (56). There is also evidence that gene expression during prenatal brain development is asymmetric (57).

Whilst genetic factors likely play a role in driving these asymmetries, they do not fully account for why these asymmetries, particularly functional asymmetries, are present at birth. One possibility relates to the idea that structural and functional connectivity precede and constrain the development of a functional region (as defined by task-based fMRI) (58). First demonstrated in the context of the visual word form area (58), there is now emerging evidence that structural and functional connectivity preceding task-based function may represent a general mechanism of cortical development $(59,60)$. Our results could represent an extension of this mechanism, whereby asymmetric functional connectivity precedes asymmetric task-based function in the auditory, visual and somatosensory networks.

Until now, studies demonstrated either leftward $(25,26)$ or rightward (23) functional asymmetry in the auditory cortex of neonates. To our knowledge, this is the first time that both leftward and rightward asymmetry in the auditory network have been demonstrated together. The marked rightward asymmetry observed in the primary auditory cortex may indicate that the ability to resolve changes in pitch, rather than temporal changes, is more important for language acquisition in the neonatal period. The leftward asymmetry of the auditory network seen in children (47) and adults (51) might then represent a developmental shift, where there is an increase in the importance of temporal information relative to pitch information for language development during childhood and adolescence. A recent finding that individuals diagnosed with autism spectrum disorder have reduced leftward asymmetry of the language network (61) suggests that disruption to this developmental shift in asymmetry may con- 
tribute to language difficulties later on.

It is well established that there is a right-hemispheric preference for face processing (62). Using electroencephalography, rightward asymmetric responses to face-like stimuli have been observed in the occipitotemporal region in neonates (63) and infants (64). Using MRI, it has also been shown that resting-state functional connectivity underlying the cortical face processing is present at birth (60), and by $4-6$ months, infants demonstrated face-selective responses in the fusiformface area $(65,66)$. These findings suggest that the rightward asymmetry of the visual network in neonates may underlie the right hemispheric specialisation of face processing seen in infancy and later life.

Both lateral sensorimotor and somatosensory networks displayed similar functional asymmetry patterns, which might relate to the somatotopic organisation of the sensorimotor cortex in neonates (67-69). Specifically, the somatosensory and lateral motor networks showed leftward asymmetry in the upper limb region of the somatotopic map (67). It has been shown that leftward lateralisation of motor network connectivity was associated with better motor performance in children (70), and that motor circuit connectivity in children diagnosed with autism spectrum disorder was more rightward asymmetric compared to children without autism spectrum disorder (71). This asymmetry may also relate to handedness, as genomic loci associated with handedness $(72,73)$ are also associated with brain asymmetry and are expressed during prenatal brain development (53). Further evidence implicating asymmetry of sensorimotor cortices with handedness comes from (46), who found that reduced leftward asymmetry of cortical thickness in the upper limb region of the postcentral gyrus was significantly associated with lefthandedness.

Not all of the functional asymmetries seen in adults (51) were present in neonates. Here, the symmetric networks were all association networks, consistent with previous work demonstrating that the maturation of RSNs in neonates follows a sequence of primary-to-association (74-77). Moreover, as these networks have been associated with higher-order cognitive processes in adults, the emergence of asymmetries in these networks may be more experience-dependent. For instance, functional asymmetry in the frontal cortex has been associated with emotional regulation $(78,79)$, reward responses (80) and cognitive set shifting (the process of consciously redirecting attention) (81); asymmetry in the visual association RSN might be related to rightward asymmetry of biological motion processing (82); and asymmetry in the temporoparietal network might relate to belief reasoning (reasoning about the content of others' mental states) (83). Further research is required to establish how functional asymmetries at birth relate to cognition and behaviour in later life. Given the marked impact that preterm birth has on cortical morphology (84-86) and functional connectivity $(75,77,87$, 88 ), it was surprising to find no differences in cortical asymmetry between preterm and term-born neonates at TEA. This is in contrast to (33), who found differences in functional asymmetry between preterm and term-born neonates at TEA.
However, that study was limited by relatively small sample sizes (33). Moreover, previous work has shown that structural asymmetries in preterm $(18)$ and term $(19,20)$ neonates are similar, and recently, (89) reported no differences in white matter asymmetry between preterm and term-born neonates at TEA. Together, these findings indicate that brain asymmetries may be robust to the effects of preterm birth, and that "multiple hits" are required to disrupt brain asymmetry (for example, preterm birth and polygenic risk) $(90,91)$. This may explain why cortical asymmetry is altered in psychiatric and neurodevelopmental disorders like schizophrenia $(7,8)$ and autism spectrum disorder $(5,6)$, which have complex and multifactorial aetiologies $(92,93)$.

Limitations. The variability in sulcal morphology between subjects, and between hemispheres, meant that the surfacebased template could not be perfectly symmetric, potentially biasing the results of the other structural and functional features. Although it was not possible to include sulcal depth asymmetry as a per-vertex explanatory variable in our main analyses due to limited compute power, we conducted a separate experiment that demonstrated this residual variability had minimal impact on the results (Figures S7 and S8).

Moreover, cortical surfaces were registered to a symmetric template using sulcal depth maps, which may have impacted the investigation of functional asymmetry, particularly in cortical regions where structural and functional correspondence is poor (94). Even though asymmetries in some association RSNs were observed here, it is possible that asymmetries in other association RSNs were not detected as a result of registering to a structural surface-based template. As such, further exploration of functional asymmetry using functional alignment is warranted, and may give insight into the development of higher order cognitive processes.

\section{Conclusion}

The structural and functional asymmetries of the neonatal cortex are likely the result of prenatal genetic factors, which are robust to early life environmental perturbations. Moreover, the asymmetric organisation of cortical structure and function at birth may constrain the development of lateralised cognitive processes and behaviours such as language and face processing.

\section{Materials and Methods}

Participants. Neonates were participants in the dHCP, approved by the National Research Ethics Committee (REC: 14/Lo/1169), and were scanned at the Evelina Newborn Imaging Centre, Evelina London Children's Hospital between 2015 and 2019. Term neonates were included if they were born between $37^{+0}$ and $41^{+6}$ weeks' GA, if they were scanned $\geq 37^{+0}$ weeks' PMA, and if both structural and functional data were available. Preterm neonates were included if they were born $<37^{+0}$ weeks' GA, if they were scanned $\geq 37^{+0}$ PMA, and if both structural and functional data were available. Both term and preterm neonates were 
excluded if their scans had incidental findings with possible/likely significance for both clinical and imaging analysis (e.g. destructive white matter lesions). However, incidental findings with possible clinical significance but unlikely analysis significance were permissible (95). Data from 442 healthy term-born neonates (200 females), and 103 pretermborn neonates (48 females) were included. Term neonates were born at $39.9 \pm 1.2$ weeks' GA, and scanned at $41.2 \pm 1.7$ weeks' PMA. Preterm neonates were born at $32.1 \pm 3.5$ weeks' GA, and scanned at $40.8 \pm 2.1$ weeks' PMA. Figure 2 shows the distribution of GA and PMA for both preterm and term neonates.

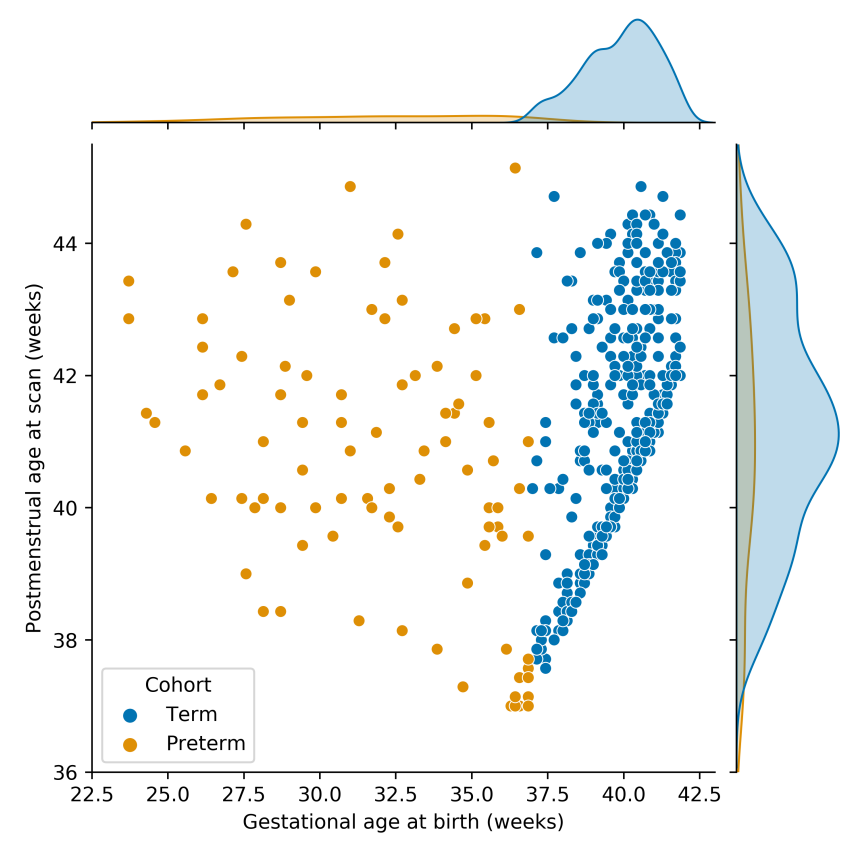

Fig. 2. Gestational age at birth and postmenstrual age at scan for preterm and term-born neonates

MRI Acquisition. MRIs were acquired in a single scan session for each neonate using a 3-Tesla Philips Achieva system (Philips Medical Systems, Best, The Netherlands). Full details regarding the preparation of neonates for scanning have been previously described (96). In brief, all neonates were scanned without sedation in a scanner environment optimised for neonatal imaging, including a dedicated 32channel neonatal coil. MR-compatible ear putty and earmuffs were used to provide additional noise attenuation. Neonates were fed, swaddled and positioned in a vacuum jacket prior to scanning to promote natural sleep. All scans were supervised by a neonatal nurse and/or paediatrician who monitored heart rate, oxygen saturation and temperature throughout the scan (96).

T2-weighted scans were acquired with a repetition time/echo time (TR/TE) of $12 \mathrm{~s} / 156 \mathrm{~ms}$, SENSE=2.11/2.58 (ax$\mathrm{ial} / \mathrm{sagittal}$ ) with in-plane resolution of $0.8 \times 0.8 \mathrm{~mm}$, slice thickness of 1.6 and overlap of $0.8 \mathrm{~mm}$. Images were motion corrected and super-resolution reconstructed resulting in $0.5 \mathrm{~mm}$ isotropic resolution (full details in (97)). fMRI scans were acquired over 15 minutes 3 seconds (2300 volumes) us- ing a multislice gradient-echo echo planar imaging sequence with multiband excitation (multiband factor 9). TR/TE was $392 \mathrm{~ms} / 38 \mathrm{~ms}$ milliseconds, flip angle was $34^{\circ}$, and the acquired spatial resolution was $2.15 \mathrm{~mm}$ isotropic $(98,99)$.

Surface Extraction. The full details of the structural pipeline are described in (100). In summary, motioncorrected, reconstructed T2-weighted images were first biascorrected and brain-extracted. Following this, images were segmented into different tissue types using the Draw-EM algorithm (100). Next, topologically correct white matter surfaces were fit first to the grey-white tissue boundary and then to the grey-white interface of the MR intensities (101). Pial surfaces were generated by expanding each white matter mesh towards the grey-cerebrospinal fluid interface $(100,101)$. Separately, an inflated surface was generated from the white surface through a process of inflation and smoothing. This inflated surface was then projected to a sphere to support surface registration. The structural pipeline generated a number of univariate features summarising cortical morphology. Of these we used: cortical thickness (estimated as the Euclidean distance between corresponding vertices on the white and pial surfaces); sulcal depth (estimates of mean surface convexity/concavity (100)); and surface area, estimated for each vertex as one third the area of each triangle it is a part of. Cortical thickness and surface area were corrected for folding bias by regressing out cortical curvature, which is the same approach as the Human Connectome Project pipeline (102).

Creating Left-Right Symmetric Surface Atlas. A prerequisite for investigating cortical asymmetry at a vertex-wise level is registration to a single template space with left-right vertex-wise correspondence. Moreover, to account for rapid morphological changes in the neonatal brain, registration to a template space should be achieved using template-totemplate registration with a spatiotemporal atlas. To achieve this, the pre-existing dHCP spatiotemporal atlas (32) was extended, both to increase the age range covered by the template to 28-44 weeks' PMA, and to enforce left-right symmetry of cortical shape (Figure 3).

This was achieved using MSM $(41,42)$ to co-register the left and right sulcal depth maps from each week of the atlas in (32). In each case, registration was run in left-to-right and right-to-left directions (replicating the approach used in (40)). Deformation maps of the left-to-right and the inverse of the right-to-left registrations were then averaged to generate an intermediate symmetric template space. Local nonsymmetric template metrics (sulcal depth, surface area and cortical thickness) and anatomical meshes (in fs_LR space; (32)) were then resampled onto their corresponding symmetric template. Finally, consecutive registrations were performed between local symmetric templates to achieve a localto-40-week PMA registration deformation (30,31). A further resampling step was then performed, to get local template metrics and anatomical meshes into 40-week PMA symmetric template space. 


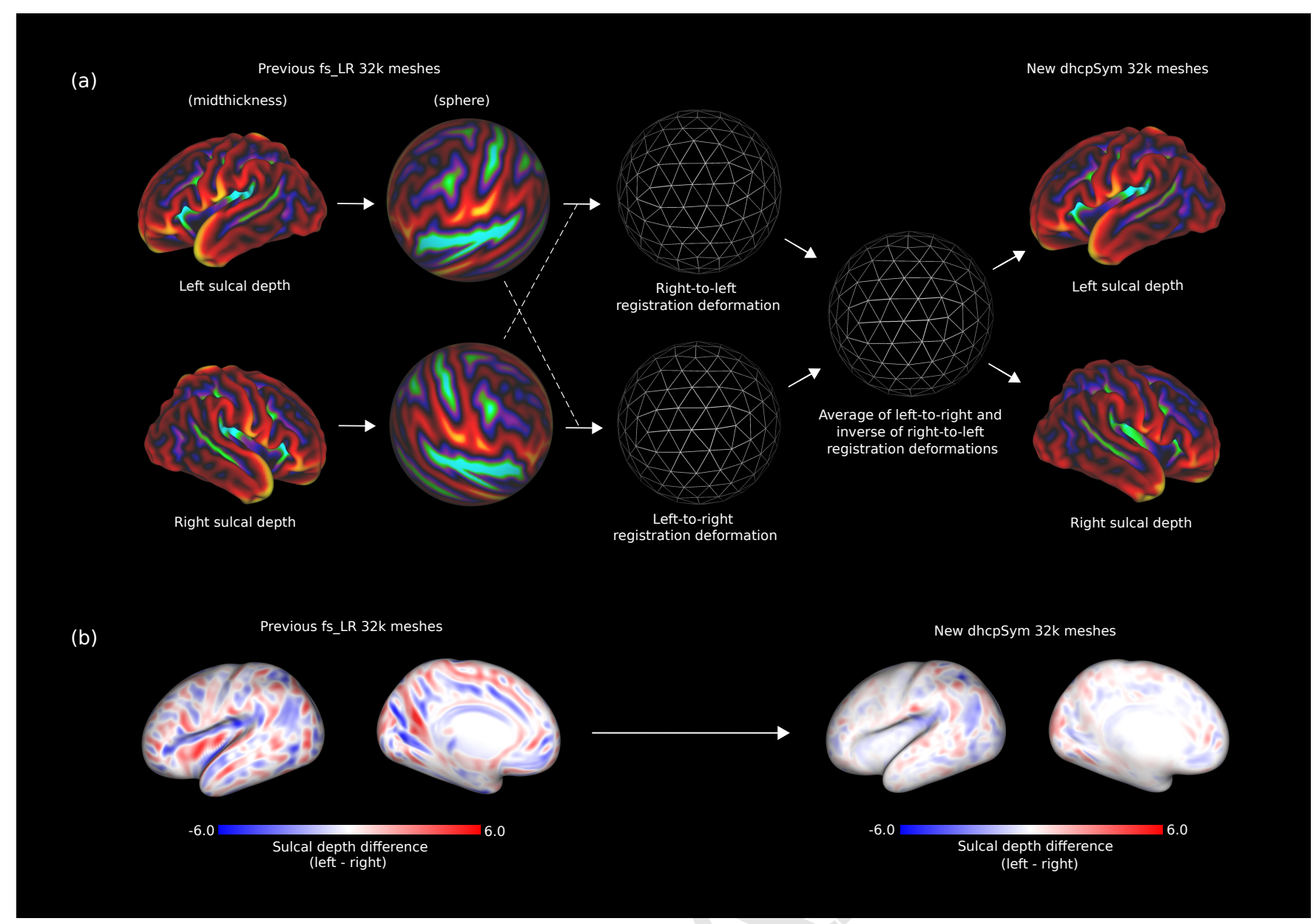

Fig. 3. (a) Development of symmetric surface-based atlas. (i) Using MSM, the left and right hemispheres from each week of the (32) atlas were co-registered in order to generate left-right vertex correspondence of coarse scale patterns of cortical folding (sulcal depth). Registration was run in left-to-right and right-to-left directions. (ii) Deformation maps of the left-toright and the inverse of the right-to-left registrations were averaged to generate an intermediate symmetric template space. (iii) Local non-symmetric template metrics and anatomical meshes were resampled into their corresponding symmetric template (dhcpSym). Consecutive registrations between local symmetric templates were then performed to achieve a local to 40-week PMA registration deformation. A further resampling step was performed to get local template metrics and anatomical meshes into 40-week PMA symmetric template space (dhcpSym40). (b) Difference between left and right 40-week PMA sulcal depth metrics for the previous non-symmetric (fs_LR) template and the new symmetric (dhcpSym) template.

Registration to Symmetric Surface Atlas. To accurately map structural asymmetry across all parts of the cortex, registration was run from all subjects' native space to their local symmetric template space, using MSM $(41,42)$, optimised for alignment of sulcal depth features. Here, MSM regularisation was reduced relative to (32) to encourage improved alignment of sulcal morphology in the frontal lobes (a region with high anatomical inter-individual variability). Subject metrics were subsequently resampled from their local template to the 40-week PMA template cortical surface, using the local-to-40 week PMA registration deformations described in Methods: Creating Left-Right Symmetric Surface Atlas.

rs-fMRI Preprocessing. We extended the dHCP functional pipeline (99) to the cortical surface by projecting individual subject rs-fMRI timeseries from the native rs-fMRI volume space to native subject surfaces warped into rs-fMRI volu- metric space, using a ribbon-constrained volume-to-surface mapping method similar to the Human Connectome Project pipeline (102). These surface rs-fMRI maps were then resampled onto the 40-week PMA symmetric surface template space using the MSM native-to-template space registration deformations. After resampling to symmetric surface space, rs-fMRI timeseries were smoothed with a kernel of $\sigma=4 \mathrm{~mm}$.

Dimensionality reduction prior to group ICA was achieved using MELODIC's Incremental Group PCA (MIGP) (103) with a dimensionality set at 2000 . Using a group ICA, dualregression approach, asymmetry observed in a dual-regressed single subject spatial map does not represent asymmetry in that individual per se, but instead represents group asymmetry that is then projected onto that individual's data. To remove this bias from the dual-regressed, single subject spatial maps, we generated symmetric group components by concatenating the left hemispheric MIGP output to the right 
hemispheric MIGP output, and vice versa. This approach was only possible because our symmetric surface template had left-right vertex correspondence. Following the left-to-right and right-to-left concatenation of the MIGP output, surfacebased group ICA was performed (set at a dimensionality of 25) (104). Group components were then dual-regressed $(105,106)$ to generate subject-level spatial maps. Of the 25 group components, 11 were labelled as signal (Figure S2). MIGP and group ICA were performed on the term neonatal cohort, and single subject spatial maps for the preterm cohort were generated by dual-regressing the term neonatal group components into the rs-fMRI timeseries of each preterm neonate scanned at TEA.

Generating Asymmetry Maps. Asymmetry maps for each cortical imaging metric (structural and functional) were generated by subtracting the right hemisphere metric from the corresponding left hemisphere metric, then normalising as $(L-R) /((L+R) / 2))$. Structural asymmetry maps were smoothed with a kernel size of $\sigma=2 \mathrm{~mm}$ to improve signalto-noise ratio, but no further smoothing of the functional asymmetry maps was performed. Individual functional asymmetry maps were then thresholded with masks that were generated by thresholding each symmetric group component at $\mathrm{Z}>3.1$. Individual functional asymmetry maps were masked after dual regression to ensure that all subject asymmetry maps for a given component had the same spatial extent, as required by FSL PALM $(107,108)$.

Statistical Analysis. Statistical analysis was performed using surface-based, vertex-wise permutation testing (FSL PALM, version alpha119 $\left.{ }^{1} ;(107,108)\right)$ with threshold-free cluster enhancement (TFCE; (109)). GA at birth, PMA at scan, biological sex, birthweight Z-score, and hemispheric volume asymmetry (normalised as $(L-R) /((L+R) / 2)$ ) were included as explanatory variables. As the time between birth and scan was variable, both GA and PMA were included as explanatory variables for the term asymmetry analysis. However, GA was removed in the preterm vs. term comparison, since the objective of this experiment was to test for the effect of age at birth. As it cannot be assumed that all term neonates are healthy, birthweight Z-score was included alongside GA as an additional, albeit imperfect, explanatory variable to account for neonatal health at birth. Birthweight Z-scores were calculated using growth curves from $(110)^{2}$, which returned birthweight Z-scores specific for GA and biological sex. Although (111) found greater cortical asymmetry in adults with larger overall brain size, total brain volume was not included as an additional explanatory variable as it is highly correlated with both GA and PMA $(32,100,112)$. Instead, we included hemispheric volume asymmetry as an additional explanatory variable, as it has been previously shown that left hemisphere has greater volume than right (15).

To investigate the impact of residual structural asymmetry on functional asymmetries, a separate analysis was per-

\footnotetext{
${ }^{1}$ https://github.com/andersonwinkler/PALM

2 http://intergrowth21.ndog.ox.ac.uk/
}

formed where individual sulcal depth asymmetry maps were included as a separate per-vertex explanatory variable ${ }^{3}$ using the -evperdat and-designperinput flags in FSL PALM (107). Due to limitations in compute power, the number of RSNs included in this analysis was limited to four.

TFCE was performed on the group average midthickness anatomical surface, with the default $H(2.0)$ and $E(0.6)$ parameters (109), which are also the same as those reported in (20). Two one-sample $t$-tests were used to investigate asymmetry in the term neonates $(\mathrm{C} 1$ : left $>$ right, and $\mathrm{C} 2$ : right $>$ left), and two two-sample unpaired $t$-tests were used to investigate differences in asymmetry between term and preterm neonates at TEA $(\mathrm{C} 1$ : term $>$ preterm, and $\mathrm{C} 2$ : preterm $>$ term). Family-wise error rate corrections were applied to $p$ values across image features and design contrasts. $p$-values were computed based on 10,000 random shuffles, with statistical significance set at $p_{\text {mcfive }}<0.05(113,114)$.

Data and Code Availability. Both the left-right symmetric, spatiotemporal cortical surface atlas ${ }^{4}$, and code used to register cortical surfaces from native space to 40 -week symmetric space $^{5}$ have been made freely available. The data and scenes used to produce the figures presented here are available from https://balsa.wustl.edu/study/L6nnx.

\section{ACKNOWLEDGEMENTS}

The authors would like to thank the participants and families recruited in the $\mathrm{dHCP}$, and all the neonatal staff at the Evelina Newborn Imaging Center, St. Thomas' Hospital, Guy's \& St. Thomas' NHS Foundation Trust, London, UK. The authors acknowledge use of the research computing facility at King's College London, Rosalind (https://rosalind.kcl.ac.uk), which is delivered in partnership with the National Institute for Health Research (NIHR) Biomedical Research Centres at South London Maudsley and Guy's St. Thomas' NHS Foundation Trusts, and part-funded by capital equipment grants from the Maudsley Charity (award 980) and Guy's St. Thomas' Charity (TR130505). The views expressed are those of the author(s) and not necessarily those of the NHS, the NIHR, King's College London, or the Department of Health and Social Care.

The dHCP project was funded by the European Research Council (ERC) under the European Union Seventh Framework Programme [FR/2007-2013]/ERC Grant Agreement no. 319,456. This study was supported in part by the Wellcome Engineering and Physical Sciences Research Council Centre for Medical Engineering at King's College London [grant WT 203,148/Z/16/Z] and the Medical Research Council (UK) [grant MR/K006355/1]. L.Z.J.W is supported by funding from the Commonwealth Scholarship Commission, United Kingdom. E.C.R is supported by an Academy of Medical Sciences/the British Heart Foundation/the Government Department of Business, Energy and Industrial Strategy/the Wellcome Trust Springboard Award [SBF003/1116] and E.C.R. and S.M.S. are supported by a Wellcome Collaborative Award [215573/Z/19/Z]. J.O. and A.D.E. received support from the Medical Research Council Centre for Neurodevelopmental Disorders, King's College London [grant MR/N026063/1]. J.O. is supported by a Sir Henry Dale Fellowship jointly funded by the Wellcome Trust and the Royal Society [grant 206,675/Z/17/Z].

\section{Bibliography}

1. Xiang-Zhen Kong, Samuel R Mathias, Tulio Guadalupe, David C Glahn, Barbara Franke, Fabrice Crivello, Nathalie Tzourio-Mazoyer, Simon E Fisher, Paul M Thompson, Clyde Francks, et al. Mapping cortical brain asymmetry in 17,141 healthy individuals worldwide via the enigma consortium. Proceedings of the National Academy of Sciences, 115(22): E5154-E5163, 2018.

2. Mathieu Vigneau, Virginie Beaucousin, Pierre-Yves Herve, Hugues Duffau, Fabrice Crivello, Olivier Houde, Bernard Mazoyer, and Nathalie Tzourio-Mazoyer. Meta-analyzing left hemisphere language areas: phonology, semantics, and sentence processing. Neuroimage, 30(4):1414-1432, 2006.

\footnotetext{
${ }^{3}$ https://fsl.fmrib.ox.ac.uk/fsl/fslwiki/PALM/ MasksAndVoxelwiseRegressors

${ }^{4}$ https: / /brain-development.org/

brain-atlases/atlases-from-the-dhcp-project/

cortical-surface-template/

${ }^{5}$ https://github.com/ecr05/dHCP_template_alignment
} 
bioRxiv preprint doi: https://doi.org/10.1101/2021.10.13.464206; this version posted October 13, 2021. The copyright holder for this preprint (which was not certified by peer review) is the author/funder, who has granted bioRxiv a license to display the preprint in perpetuity. It is made available under aCC-BY-NC-ND 4.0 International license.

3. Bernard Mazoyer, Laure Zago, Gaël Jobard, Fabrice Crivello, Marc Joliot, Guy Perchey, Emmanuel Mellet, Laurent Petit, and Nathalie Tzourio-Mazoyer. Gaussian mixture modeling of hemispheric lateralization for language in a large sample of healthy individuals balanced for handedness. PloS one, 9(6):e101165, 2014.

4. Zonglei Zhen, Zetian Yang, Lijie Huang, Xiang-zhen Kong, Xu Wang, Xiaobin Dang, Yangyue Huang, Yiying Song, and Jia Liu. Quantifying interindividual variability and asymmetry of face-selective regions: a probabilistic functional atlas. Neuroimage, 113:13-25, 2015.

5. Merel C Postema, Daan Van Rooij, Evdokia Anagnostou, Celso Arango, Guillaume Auzias, Marlene Behrmann, Geraldo Busatto Filho, Sara Calderoni, Rosa Calvo, Eileen Daly, et al. Altered structural brain asymmetry in autism spectrum disorder in a study of 54 datasets. Nature communications, 10(1):1-12, 2019.

6. Lisa T Eyler, Karen Pierce, and Eric Courchesne. A failure of left temporal cortex to specialize for language is an early emerging and fundamental property of autism. Brain, 135 (3):949-960, 2012.

7. Yasuhiro Kawasaki, Michio Suzuki, Tutomu Takahashi, Shigeru Nohara, Philip K McGuire, Hikaru Seto, and Masayoshi Kurachi. Anomalous cerebral asymmetry in patients with schizophrenia demonstrated by voxel-based morphometry. Biological psychiatry, 63(8): 793-800, 2008.

8. Katherine SF Damme, Teresa Vargas, Vince Calhoun, Jessica Turner, and Vijay A Mittal. Global and specific cortical volume asymmetries in individuals with psychosis risk syndrome and schizophrenia: A mixed cross-sectional and longitudinal perspective. Schizophrenia bulletin, 46(3):713-721, 2020.

9. Xiang-Zhen Kong, Premika SW Boedhoe, Yoshinari Abe, Pino Alonso, Stephanie H Ameis, Paul D Arnold, Francesca Assogna, Justin T Baker, Marcelo C Batistuzzo, Francesco Benedetti, et al. Mapping cortical and subcortical asymmetry in obsessive-compulsive disorder: findings from the enigma consortium. Biological Psychiatry, 87(12):1022-1034, 2020.

10. Merel Postema, Martine Hoogman, Sara Ambrosino, Philip Asherson, Tobias Banaschewski, Cibele E Bandeira, Alexandr Baranov, Claiton HD Bau, Sarah Baumeister, Ramona Baur-Streubel, et al. Analysis of structural brain asymmetries in attentiondeficit/hyperactivity disorder in 39 datasets. Journal of Child Psychology and Psychiatry, 2021.

11. Irene Altarelli, François Leroy, Karla Monzalvo, Joel Fluss, Catherine Billard, Ghislaine Dehaene-Lambertz, Albert M Galaburda, and Franck Ramus. Planum temporale asymmetry in developmental dyslexia: Revisiting an old question. Human brain mapping, 35 (12):5717-5735, 2014

12. Onur Güntürkün and Sebastian Ocklenburg. Ontogenesis of lateralization. Neuron, 94(2): 249-263, 2017

13. Dorothy VM Bishop. Cerebral asymmetry and language development: cause, correlate, or consequence? Science, 340(6138), 2013.

14. Véronique Duboc, Pascale Dufourcq, Patrick Blader, and Myriam Roussigné. Asymmetry of the brain: development and implications. Annual review of genetics, 49:647-672, 2015.

15. John H Gilmore, Weili Lin, Marcel W Prastawa, Christopher B Looney, Y Sampath K Vetsa, Rebecca C Knickmeyer, Dianne D Evans, J Keith Smith, Robert M Hamer, Jeffrey A Lieberman, et al. Regional gray matter growth, sexual dimorphism, and cerebral asymmetry in the neonatal brain. Journal of Neuroscience, 27(6):1255-1260, 2007.

16. Gregor Kasprian, Georg Langs, Peter $C$ Brugger, Mario Bittner, Michael Weber, Mavilde Arantes, and Daniela Prayer. The prenatal origin of hemispheric asymmetry: an in utero neuroimaging study. Cerebral cortex, 21(5):1076-1083, 2011.

17. Piotr A Habas, Julia A Scott, Ahmad Roosta, Vidya Rajagopalan, Kio Kim, Francois Rousseau, A James Barkovich, Orit A Glenn, and Colin Studholme. Early folding patterns and asymmetries of the normal human brain detected from in utero mri. Cerebral cortex, 22(1):13-25, 2012

18. Jessica Dubois, M Benders, François Lazeyras, Cristina Borradori-Tolsa, R Ha-Vinh Leuchter, Jean-François Mangin, and Petra S Hüppi. Structural asymmetries of perisylvian regions in the preterm newborn. Neuroimage, 52(1):32-42, 2010.

19. Gang Li, Jingxin Nie, Li Wang, Feng Shi, Amanda E Lyall, Weili Lin, John H Gilmore, and Dinggang Shen. Mapping longitudinal hemispheric structural asymmetries of the human cerebral cortex from birth to 2 years of age. Cerebral cortex, 24(5):1289-1300, 2014

20. Jason Hill, Donna Dierker, Jeffrey Neil, Terrie Inder, Andrew Knutsen, John Harwell, Timothy Coalson, and David Van Essen. A surface-based analysis of hemispheric asymmetries and folding of cerebral cortex in term-born human infants. Journal of neuroscience, 30(6): 2268-2276, 2010

21. H Glasel, François Leroy, Jessica Dubois, Lucie Hertz-Pannier, Jean-François Mangin, and Ghislaine Dehaene-Lambertz. A robust cerebral asymmetry in the infant brain: the rightward superior temporal sulcus. Neuroimage, 58(3):716-723, 2011.

22. Jessica Dubois, Lucie Hertz-Pannier, A Cachia, JF Mangin, D Le Bihan, and Ghislaine Dehaene-Lambertz. Structural asymmetries in the infant language and sensori-motor networks. Cerebral cortex, 19(2):414-423, 2009.

23. Daniela Perani, Maria Cristina Saccuman, Paola Scifo, Danilo Spada, Guido Andreolli, Rosanna Rovelli, Cristina Baldoli, and Stefan Koelsch. Functional specializations for music processing in the human newborn brain. Proceedings of the National Academy of Sciences, 107(10):4758-4763, 2010.

24. Daniela Perani, Maria C Saccuman, Paola Scifo, Alfred Anwander, Danilo Spada, Cristina Baldoli, Antonella Poloniato, Gabriele Lohmann, and Angela D Friederici. Neural language networks at birth. Proceedings of the National Academy of Sciences, 108(38):1605616061, 2011

25. Ghislaine Dehaene-Lambertz, Stanislas Dehaene, and Lucie Hertz-Pannier. Functional neuroimaging of speech perception in infants. science, 298(5600):2013-2015, 2002.

26. Ghislaine Dehaene-Lambertz, Lucie Hertz-Pannier, Jessica Dubois, Sébastien Mériaux, Alexis Roche, Mariano Sigman, and Stanislas Dehaene. Functional organization of perisylvian activation during presentation of sentences in preverbal infants. Proceedings of the National Academy of Sciences, 103(38):14240-14245, 2006.

27. Alan Tucholka, Virgile Fritsch, Jean-Baptiste Poline, and Bertrand Thirion. An empirical comparison of surface-based and volume-based group studies in neuroimaging. Neuroim- age, 63(3):1443-1453, 2012

28. Timothy S Coalson, David C Van Essen, and Matthew F Glasser. The impact of traditional neuroimaging methods on the spatial localization of cortical areas. Proceedings of the National Academy of Sciences, 115(27):E6356-E6365, 2018.

29. Stephen M Smith, Christian F Beckmann, Jesper Andersson, Edward J Auerbach, Janine Bijsterbosch, Gwenaëlle Douaud, Eugene Duff, David A Feinberg, Ludovica Griffanti, Michael P Harms, et al. Resting-state fmri in the human connectome project. Neuroimage, 80:144-168, 2013

30. Ahmed Serag, Paul Aljabar, Serena Counsell, James Boardman, Jo V Hajnal, and Daniel Rueckert. Lisa: Longitudinal image registration via spatio-temporal atlases. In 2012 9th IEEE International Symposium on Biomedical Imaging (ISBI), pages 334-337. IEEE, 2012.

31. Andreas Schuh, Antonios Makropoulos, Emma C Robinson, Lucilio Cordero-Grande, Emer Hughes, Jana Hutter, Anthony N Price, Maria Murgasova, Rui Pedro AG Teixeira, Nora Tusor, et al. Unbiased construction of a temporally consistent morphological atlas of neonatal brain development. bioRxiv, page 251512, 2018.

32. Jelena Bozek, Antonios Makropoulos, Andreas Schuh, Sean Fitzgibbon, Robert Wright, Matthew F Glasser, Timothy S Coalson, Jonathan O'Muircheartaigh, Jana Hutter, Anthony N Price, et al. Construction of a neonatal cortical surface atlas using multimodal surface matching in the developing human connectome project. Neurolmage, 179:11-29, 2018.

33. Soo Hyun Kwon, Dustin Scheinost, Cheryl Lacadie, Gordon Sze, Karen C Schneider, Feng Dai, R Todd Constable, and Laura R Ment. Adaptive mechanisms of developing brain: cerebral lateralization in the prematurely-born. Neuroimage, 108:144-150, 2015.

34. Joseph J Volpe. Dysmaturation of premature brain: importance, cellular mechanisms, and potential interventions. Pediatric neurology, 95:42-66, 2019.

35. JP Boardman and SJ Counsell. Invited review: factors associated with atypical brain development in preterm infants: insights from magnetic resonance imaging. Neuropathology and applied neurobiology, 46(5):413-421, 2020.

36. Melinda McBryde, Grace C Fitzallen, Helen G Liley, H Gerry Taylor, and Samudragupta Bora. Academic outcomes of school-aged children born preterm: A systematic review and meta-analysis. JAMA network open, 3(4):e202027-e202027, 2020.

37. Christopher R Brydges, Jasmin K Landes, Corinne L Reid, Catherine Campbell, Noel French, and Mike Anderson. Cognitive outcomes in children and adolescents born very preterm: a meta-analysis. Developmental Medicine \& Child Neurology, 60(5):452-468, 2018.

38. E Sabrina Twilhaar, Jorrit F de Kieviet, Cornelieke SH Aarnoudse-Moens, Ruurd M van Elburg, and Jaap Oosterlaan. Academic performance of children born preterm: a metaanalysis and meta-regression. Archives of Disease in Childhood-Fetal and Neonatal Edition, 103(4):F322-F330, 2018.

39. Vanessa N Kovachy, Jenna N Adams, John S Tamaresis, and Heidi M Feldman. Reading abilities in school-aged preterm children: a review and meta-analysis. Developmental Medicine \& Child Neurology, 57(5):410-419, 2015.

40. Kara E Garcia, Emma C Robinson, Dimitrios Alexopoulos, Donna L Dierker, Matthew F Glasser, Timothy S Coalson, Cynthia M Ortinau, Daniel Rueckert, Larry A Taber, David C Van Essen, et al. Dynamic patterns of cortical expansion during folding of the preterm human brain. Proceedings of the National Academy of Sciences, 115(12):3156-3161, 2018.

41. Emma C Robinson, Saad Jbabdi, Matthew F Glasser, Jesper Andersson, Gregory C Burgess, Michael P Harms, Stephen M Smith, David C Van Essen, and Mark Jenkinson. Msm: a new flexible framework for multimodal surface matching. Neuroimage, 100: 414-426, 2014

42. Emma C Robinson, Kara Garcia, Matthew F Glasser, Zhengdao Chen, Timothy S Coalson, Antonios Makropoulos, Jelena Bozek, Robert Wright, Andreas Schuh, Matthew Webster, et al. Multimodal surface matching with higher-order smoothness constraints. Neuroimage, 167:453-465, 2018.

43. Jessica Dubois, Manon Benders, Arnaud Cachia, Francois Lazeyras, $\mathrm{R}$ Ha-Vinh Leuchter, Stéphane V Sizonenko, Cristina Borradori-Tolsa, Jean-François Mangin, and Petra Susan Hüppi. Mapping the early cortical folding process in the preterm newborn brain. Cerebral cortex, 18(6):1444-1454, 2008.

44. François Leroy, Qing Cai, Stephanie L Bogart, Jessica Dubois, Olivier Coulon, Karla Monzalvo, Clara Fischer, Hervé Glasel, Lise Van der Haegen, Audrey Bénézit, et al. New human-specific brain landmark: the depth asymmetry of superior temporal sulcus. Proceedings of the National Academy of Sciences, 112(4):1208-1213, 2015.

45. David C Van Essen. A population-average, landmark-and surface-based (pals) atlas of human cerebral cortex. Neuroimage, 28(3):635-662, 2005.

46. Zhiqiang Sha, Antonietta Pepe, Dick Schijven, Amaia Carrion Castillo, James M Roe, René Westerhausen, Marc Joliot, Simon E Fisher, Fabrice Crivello, and Clyde Francks. Lefthandedness and its genetic influences are associated with structural asymmetries mapped across the cerebral cortex in 31,864 individuals. bioRxiv, 2021.

47. Amanda G Wood, A Simon Harvey, R Mark Wellard, DF Abbott, V Anderson, M Kean, MM Saling, and Graeme D Jackson. Language cortex activation in normal children. Neurology, 63(6):1035-1044, 2004

48. Robert $\mathrm{J}$ Zatorre and Pascal Belin. Spectral and temporal processing in human auditory cortex. Cerebral cortex, 11(10):946-953, 2001.

49. Robert J Zatorre, Pascal Belin, and Virginia B Penhune. Structure and function of auditory cortex: music and speech. Trends in cognitive sciences, 6(1):37-46, 2002.

50. Krista L Hyde, Isabelle Peretz, and Robert J Zatorre. Evidence for the role of the right auditory cortex in fine pitch resolution. Neuropsychologia, 46(2):632-639, 2008.

51. O Agcaoglu, R Miller, Andy R Mayer, Kenneth Hugdahl, and Vince D Calhoun. Lateralization of resting state networks and relationship to age and gender. Neuroimage, 104: 310-325, 2015

52. Hesheng Liu, Steven M Stufflebeam, Jorge Sepulcre, Trey Hedden, and Randy L Buckner. Evidence from intrinsic activity that asymmetry of the human brain is controlled by multiple factors. Proceedings of the National Academy of Sciences, 106(48):20499-20503, 2009.

53. Zhiqiang Sha, Dick Schijven, Amaia Carrion-Castillo, Marc Joliot, Bernard Mazoyer, Simon Fisher, Fabrice Crivello, and Clyde Francks. The genetic architecture of structural left-right 
bioRxiv preprint doi: https://doi.org/10.1101/2021.10.13.464206; this version posted October 13, 2021. The copyright holder for this preprint (which was not certified by peer review) is the author/funder, who has granted bioRxiv a license to display the preprint in perpetuity. It is made available under aCC-BY-NC-ND 4.0 International license.

asymmetry of the human brain. bioRxiv, 2020.

54. Sam Horng, Gabriel Kreiman, Charlene Ellsworth, Damon Page, Marissa Blank, Kathleen Millen, and Mriganka Sur. Differential gene expression in the developing lateral geniculate nucleus and medial geniculate nucleus reveals novel roles for zic4 and foxp2 in visual and auditory pathway development. Journal of Neuroscience, 29(43):13672-13683, 2009.

55. Isabel Del Pino, Chiara Tocco, Elia Magrinelli, Andrea Marcantoni, Celeste Ferraguto, Giulia Tomagra, Michele Bertacchi, Christian Alfano, Xavier Leinekugel, Andreas Frick, et al. Coup-tfi/nr2f1 orchestrates intrinsic neuronal activity during development of the somatosensory cortex. Cerebral Cortex, 30(11):5667-5685, 2020.

56. Matthew L O'Sullivan, Joris de Wit, Jeffrey N Savas, Davide Comoletti, Stefanie Otto-Hitt, John R Yates III, and Anirvan Ghosh. Flrt proteins are endogenous latrophilin ligands and regulate excitatory synapse development. Neuron, 73(5):903-910, 2012.

57. Tao Sun, Christina Patoine, Amir Abu-Khalil, Jane Visvader, Eleanor Sum, Timothy J Cherry, Stuart H Orkin, Daniel H Geschwind, and Christopher A Walsh. Early asymmetry of gene transcription in embryonic human left and right cerebral cortex. Science, 308 (5729):1794-1798, 2005

58. Zeynep M Saygin, David E Osher, Elizabeth S Norton, Deanna A Youssoufian, Sara D Beach, Jenelle Feather, Nadine Gaab, John DE Gabrieli, and Nancy Kanwisher. Connectivity precedes function in the development of the visual word form area. Nature neuroscience, 19(9):1250-1255, 2016

59. Jin Li, David E Osher, Heather A Hansen, and Zeynep M Saygin. Innate connectivity patterns drive the development of the visual word form area. Scientific reports, 10(1) $1-12,2020$.

60. Frederik S Kamps, Cassandra L Hendrix, Patricia A Brennan, and Daniel D Dilks. Connectivity at the origins of domain specificity in the cortical face and place networks. Proceedings of the National Academy of Sciences, 117(11):6163-6169, 2020.

61. Olessia Jouravlev, Alexander JE Kell, Zachary Mineroff, Amanda J Haskins, Dima Ayyash, Nancy Kanwisher, and Evelina Fedorenko. Reduced language lateralization in autism and the broader autism phenotype as assessed with robust individual-subjects analyses. Autism Research, 13(10):1746-1761, 2020.

62. Gregory McCarthy, Aina Puce, John C Gore, and Truett Allison. Face-specific processing in the human fusiform gyrus. Journal of cognitive neuroscience, 9(5):605-610, 1997.

63. Marco Buiatti, Elisa Di Giorgio, Manuela Piazza, Carlo Polloni, Giuseppe Menna, Fabrizio Taddei, Ermanno Baldo, and Giorgio Vallortigara. Cortical route for facelike pattern processing in human newborns. Proceedings of the National Academy of Sciences, 116(10): 4625-4630, 2019

64. Parvaneh Adibpour, Jessica Dubois, and Ghislaine Dehaene-Lambertz. Right but not left hemispheric discrimination of faces in infancy. Nature Human Behaviour, 2(1):67-79, 2018

65. Heather Kosakowski, Michael Cohen, Atsushi Takahashi, Boris Keil, Nancy Kanwisher, and Rebecca Saxe. Selective responses to faces, scenes, and bodies in the ventral visual pathway of infants. 2021

66. Ben Deen, Hilary Richardson, Daniel D Dilks, Atsushi Takahashi, Boris Keil, Lawrence L Wald, Nancy Kanwisher, and Rebecca Saxe. Organization of high-level visual cortex in human infants. Nature communications, 8(1):1-10, 2017.

67. S Dall'Orso, J Steinweg, AG Allievi, AD Edwards, E Burdet, and T Arichi. Somatotopic mapping of the developing sensorimotor cortex in the preterm human brain. Cerebral cortex, 28(7):2507-2515, 2018.

68. Tomoki Arichi, A Moraux, A Melendez, Valentina Doria, M Groppo, Nazakat Merchant, S Combs, Etienne Burdet, David J Larkman, Serena J Counsell, et al. Somatosensory cortical activation identified by functional $\mathrm{mri}$ in preterm and term infants. Neuroimage, 49 (3):2063-2071, 2010.

69. Alessandro G Allievi, Tomoki Arichi, Nora Tusor, Jessica Kimpton, Sophie Arulkumaran, Serena J Counsell, A David Edwards, and Etienne Burdet. Maturation of sensori-motor functional responses in the preterm brain. Cerebral Cortex, 26(1):402-413, 2016.

70. Anita D Barber, Priti Srinivasan, Suresh E Joel, Brian S Caffo, James J Pekar, and Stewart H Mostofsky. Motor "dexterity"? Evidence that left hemisphere lateralization of moto circuit connectivity is associated with better motor performance in children. Cerebral Cor tex, 22(1):51-59, 2012.

71. Dorothea L Floris, Anita D Barber, Mary Beth Nebel, Mary Martinelli, Meng-Chuan Lai, Deana Crocetti, Simon Baron-Cohen, John Suckling, James J Pekar, and Stewart H Mostofsky. Atypical lateralization of motor circuit functional connectivity in children with autism is associated with motor deficits. Molecular autism, 7(1):1-14, 2016.

72. Akira Wiberg, Michael $\mathrm{Ng}$, Yasser Al Omran, Fidel Alfaro-Almagro, Paul McCarthy, Jonathan Marchini, David L Bennett, Stephen Smith, Gwenaëlle Douaud, and Dominic Furniss. Handedness, language areas and neuropsychiatric diseases: insights from brain imaging and genetics. Brain, 142(10):2938-2947, 2019.

73. Gabriel Cuellar-Partida, Joyce Y Tung, Nicholas Eriksson, Eva Albrecht, Fazil Aliev, Ole A Andreassen, Inês Barroso, Jacques S Beckmann, Marco P Boks, Dorret I Boomsma, et al. Genome-wide association study identifies 48 common genetic variants associated with handedness. Nature human behaviour, 5(1):59-70, 2021.

74. Valentina Doria, Christian F Beckmann, Tomoki Arichi, Nazakat Merchant, Michela Groppo, Federico E Turkheimer, Serena J Counsell, Maria Murgasova, Paul Aljabar, Rita G Nunes, et al. Emergence of resting state networks in the preterm human brain. Proceedings of the National Academy of Sciences, 107(46):20015-20020, 2010

75. Christopher D Smyser, Terrie E Inder, Joshua S Shimony, Jason E Hill, Andrew J Degnan, Abraham Z Snyder, and Jeffrey J Neil. Longitudinal analysis of neural network development in preterm infants. Cerebral cortex, 20(12):2852-2862, 2010.

76. Peter Fransson, Beatrice Skiöld, Sandra Horsch, Anders Nordell, Mats Blennow, Hugo Lagercrantz, and Ulrika Åden. Resting-state networks in the infant brain. Proceedings of the National Academy of Sciences, 104(39):15531-15536, 2007.

77. Michael Eyre, Sean P Fitzgibbon, Judit Ciarrusta, Lucilio Cordero-Grande, Anthony N Price, Tanya Poppe, Andreas Schuh, Emer Hughes, Camilla O'keeffe, Jakki Brandon, et al. The developing human connectome project: typical and disrupted perinatal functional connectivity. BioRxiv, 2020.

78. Mai Uchida, Joseph Biederman, John DE Gabrieli, Jamie Micco, Carlo de Los Angeles,
Ariel Brown, Tara Kenworthy, Elana Kagan, and Susan Whitfield-Gabrieli. Emotion regulation ability varies in relation to intrinsic functional brain architecture. Social cognitive and affective neuroscience, 10(12):1738-1748, 2015.

79. John D Herrington, Wendy Heller, Aprajita Mohanty, Anna S Engels, Marie T Banich, An drew G Webb, and Gregory A Miller. Localization of asymmetric brain function in emotion and depression. Psychophysiology, 47(3):442-454, 2010.

80. Alizée Lopez-Persem, Léa Roumazeilles, Davide Folloni, Kévin Marche, Elsa F Fourag nan, Nima Khalighinejad, Matthew FS Rushworth, and Jérôme Sallet. Differential functional connectivity underlying asymmetric reward-related activity in human and nonhuman primates. Proceedings of the National Academy of Sciences, 117(45):28452-28462, 2020

81. Seiki Konishi, Toshihiro Hayashi, Idai Uchida, Hideyuki Kikyo, Emi Takahashi, and Yasush Miyashita. Hemispheric asymmetry in human lateral prefrontal cortex during cognitive set shifting. Proceedings of the National Academy of Sciences, 99(11):7803-7808, 2002.

82. Emily Grossman, Michael Donnelly, R Price, D Pickens, V Morgan, G Neighbor, and Randolph Blake. Brain areas involved in perception of biological motion. Journal of cognitive neuroscience, 12(5):711-720, 2000.

83. Katrin Döhnel, Tobias Schuwerk, Jörg Meinhardt, Beate Sodian, Göran Hajak, and Monika Sommer. Functional activity of the right temporo-parietal junction and of the medial prefrontal cortex associated with true and false belief reasoning. Neuroimage, 60(3):16521661,2012

84. Erin Engelhardt, Terrie E Inder, Dimitrios Alexopoulos, Donna L Dierker, Jason Hill, David Van Essen, and Jeffrey J Neil. Regional impairments of cortical folding in premature infants. Annals of neurology, 77(1):154-162, 2015.

85. M Ajayi-Obe, N Saeed, FM Cowan, MA Rutherford, and AD Edwards. Reduced develop ment of cerebral cortex in extremely preterm infants. The Lancet, 356(9236):1162-1163, 2000.

86. Ralica Dimitrova, Maximilian Pietsch, Judit Ciarrusta, Sean P. Fitzgibbon, Logan Z.J. Williams, Daan Christiaens, Lucilio Cordero-Grande, Dafnis Batalle, Antonios Makropoulos, Andreas Schuh, Anthony N. Price, Jana Hutter, Rui PAG Teixeira, Emer Hughes, Andrew Chew, Shona Falconer, Olivia Carney, Alexia Egloff, J-Donald Tournier, Grainne McAlonan, Mary A. Rutherford, Serena J. Counsell, Emma C. Robinson, Joseph V. Hajnal, Daniel Rueckert, A. David Edwards, and Jonathan O'Muircheartaigh. Preterm birth alters the development of cortical microstructure and morphology at term-equivalent age. $\mathrm{Neu}$ rolmage, 243:118488, 2021. ISSN 1053-8119. doi: https://doi.org/10.1016/j.neuroimage. 2021.118488

87. Gareth Ball, Paul Aljabar, Tomoki Arichi, Nora Tusor, D Cox, Nazakat Merchant, P Nongena, Joseph V Hajnal, A David Edwards, and Serena J Counsell. Machine-learning to characterise neonatal functional connectivity in the preterm brain. Neuroimage, 124:267275,2016

88. Christopher D Smyser, Abraham Z Snyder, Joshua S Shimony, Anish Mitra, Terrie E Inder, and Jeffrey $\mathrm{J}$ Neil. Resting-state network complexity and magnitude are reduced in prematurely born infants. Cerebral cortex, 26(1):322-333, 2016.

89. Tingting Liu, Fusheng Gao, Weihao Zheng, Yuqing You, Zhiyong Zhao, Ying Lv, Weijun Chen, Hongxi Zhang, Chai Ji, and Dan Wu. Diffusion mri of the infant brain reveals unique asymmetry patterns during the first-half-year of development. Neurolmage, page 118465 , 2021

90. Wolfgang Gaebel and Jürgen Zielasek. Integrative etiopathogenetic models of psychotic disorders: methods, evidence and concepts. Schizophrenia bulletin, 37(suppl 2):S5-S12, 2011.

91. Celso Arango, Covadonga M Díaz-Caneja, Patrick D McGorry, Judith Rapoport, Iris E Sommer, Jacob A Vorstman, David McDaid, Oscar Marín, Elena Serrano-Drozdowskyj, Robert Freedman, et al. Preventive strategies for mental health. The Lancet Psychiatry, 5 (7):591-604, 2018

92. Justin Davis, Harris Eyre, Felice N Jacka, Seetal Dodd, Olivia Dean, Sarah McEwen, Monojit Debnath, John McGrath, Michael Maes, Paul Amminger, et al. A review of vulnerability and risks for schizophrenia: beyond the two hit hypothesis. Neuroscience \& Biobehavioral Reviews, 65:185-194, 2016.

93. Craig J Newschaffer, Lisa A Croen, Julie Daniels, Ellen Giarelli, Judith K Grether, Susan E Levy, David S Mandell, Lisa A Miller, Jennifer Pinto-Martin, Judy Reaven, et al. The epidemiology of autism spectrum disorders. Annu. Rev. Public Health, 28:235-258, 2007.

94. Matthew F Glasser, Timothy S Coalson, Emma C Robinson, Carl D Hacker, John Harwell, Essa Yacoub, Kamil Ugurbil, Jesper Andersson, Christian F Beckmann, Mark Jenkinson, et al. A multi-modal parcellation of human cerebral cortex. Nature, 536(7615):171-178, 2016

95. Olivia Carney, Emer Hughes, Nora Tusor, Ralica Dimitrova, Sophie Arulkumaran, Kelly Pegoretti Baruteau, Alexia Egloff Collado, Lucilio Cordero-Grande, Andrew Chew, Shona Falconer, et al. Incidental findings on brain mr imaging of asymptomatic term neonates in the developing human connectome project. EClinicalMedicine, 38:100984, 2021

96. Emer J Hughes, Tobias Winchman, Francesco Padormo, Rui Teixeira, Julia Wurie Maryanne Sharma, Matthew Fox, Jana Hutter, Lucilio Cordero-Grande, Anthony N Price, et al. A dedicated neonatal brain imaging system. Magnetic resonance in medicine, 78(2): 794-804, 2017

97. Lucilio Cordero-Grande, Emer J Hughes, Jana Hutter, Anthony N Price, and Joseph V Hajnal. Three-dimensional motion corrected sensitivity encoding reconstruction for multi-sho multi-slice mri: application to neonatal brain imaging. Magnetic resonance in medicine, 79 (3):1365-1376, 2018.

98. A Price, Lucilio Cordero-Grande, S Malik, Maryam Abaei, Tomoki Arichi, Emer J Hughes, Daniel Rueckert, A David Edwards, and Joseph V Hajnal. Accelerated neonatal fmri using multiband epi. In Proceedings of the 23rd Annual Meeting of ISMRM, Toronto, Canada, page 3911, 2015.

99. Sean Patrick Fitzgibbon, Samuel J Harrison, Mark Jenkinson, Luke Baxter, Emma Claire Robinson, Matteo Bastiani, Jelena Bozek, Vyacheslav Karolis, Lucilio Cordero Grande, Anthony N Price, et al. The developing human connectome project (dhcp) automated resting-state functional processing framework for newborn infants. BioRxiv, page 766030 , 2020.

100. Antonios Makropoulos, Emma C Robinson, Andreas Schuh, Robert Wright, Sean Fitzgib- 
bioRxiv preprint doi: https://doi.org/10.1101/2021.10.13.464206; this version posted October 13, 2021. The copyright holder for this preprint (which was not certified by peer review) is the author/funder, who has granted bioRxiv a license to display the preprint in perpetuity. It is made available under aCC-BY-NC-ND 4.0 International license.

bon, Jelena Bozek, Serena J Counsell, Johannes Steinweg, Katy Vecchiato, Jonathan Passerat-Palmbach, et al. The developing human connectome project: A minimal processing pipeline for neonatal cortical surface reconstruction. Neuroimage, 173:88-112, 2018.

101. Andreas Schuh, Antonios Makropoulos, Robert Wright, Emma C Robinson, Nora Tusor Johannes Steinweg, Emer Hughes, Lucilio Cordero Grande, Anthony Price, Jana Hutter et al. A deformable model for the reconstruction of the neonatal cortex. In 2017 IEEE 14th International Symposium on Biomedical Imaging (ISBI 2017), pages 800-803. IEEE, 2017.

102. Matthew F Glasser, Stamatios N Sotiropoulos, J Anthony Wilson, Timothy S Coalson, Bruce Fischl, Jesper L Andersson, Junqian Xu, Saad Jbabdi, Matthew Webster, Jonathan R Polimeni, et al. The minimal preprocessing pipelines for the human connectome project. Neuroimage, 80:105-124, 2013.

103. Stephen M Smith, Aapo Hyvärinen, Gaël Varoquaux, Karla L Miller, and Christian F Beckmann. Group-pca for very large fmri datasets. Neuroimage, 101:738-749, 2014.

104. Christian F Beckmann and Stephen M Smith. Tensorial extensions of independent component analysis for multisubject fmri analysis. Neuroimage, 25(1):294-311, 2005.

105. Christian F Beckmann, Clare E Mackay, Nicola Filippini, Stephen M Smith, et al. Group comparison of resting-state fmri data using multi-subject ica and dual regression. Neuroimage, 47(Suppl 1):S148, 2009.

106. Lisa D Nickerson, Stephen M Smith, Döst Öngür, and Christian F Beckmann. Using dual regression to investigate network shape and amplitude in functional connectivity analyses. Frontiers in neuroscience, 11:115, 2017.

107. Anderson M Winkler, Gerard R Ridgway, Matthew A Webster, Stephen M Smith, and Thomas E Nichols. Permutation inference for the general linear model. Neuroimage, 92:381-397, 2014

108. Anderson M Winkler, Gerard R Ridgway, Gwenaëlle Douaud, Thomas E Nichols, and Stephen M Smith. Faster permutation inference in brain imaging. Neuroimage, 141:502516, 2016

109. Stephen M Smith and Thomas E Nichols. Threshold-free cluster enhancement: addressing problems of smoothing, threshold dependence and localisation in cluster inference. Neuroimage, 44(1):83-98, 2009.

110. José Villar, Leila Cheikh Ismail, Cesar G Victora, Eric O Ohuma, Enrico Bertino, Doug G Altman, Ann Lambert, Aris T Papageorghiou, Maria Carvalho, Yasmin A Jaffer, et al. International standards for newborn weight, length, and head circumference by gestational age and sex: the newborn cross-sectional study of the intergrowth-21st project. The Lancet, 384(9946):857-868, 2014.

111. Xiaojian Kang, Timothy J Herron, Marc Ettlinger, and David L Woods. Hemispheric asymmetries in cortical and subcortical anatomy. Laterality: Asymmetries of Body, Brain and Cognition, 20(6):658-684, 2015

112. Ralica Dimitrova, Sophie Arulkumaran, Olivia Carney, Andrew Chew, Shona Falconer, Judit Ciarrusta, Thomas Wolfers, Dafnis Batalle, Lucilio Cordero-Grande, Anthony N Price, et al. Phenotyping the preterm brain: characterising individual deviations from normative volumetric development in two large infant cohorts. Cerebral Cortex, pages 2020-08, 2021.

113. Bianca AV Alberton, Thomas E Nichols, Humberto R Gamba, and Anderson M Winkler. Multiple testing correction overcontrasts for brain imaging. Neurolmage, page 116760 , 2020.

114. Anderson M Winkler, Matthew A Webster, Jonathan C Brooks, Irene Tracey, Stephen M Smith, and Thomas E Nichols. Non-parametric combination and related permutation tests for neuroimaging. Human brain mapping, 37(4):1486-1511, 2016. 